\title{
COMMUNICATION
}

\section{Chirurgie implantaire guidée : acquisition, planification, modélisation et production d'un guide chirurgical. Mise en place d'une chaine numérique pour la création interne et l'utilisation de guides chirurgicaux}

\author{
T. Pajot (Paris), S. Ketoff (Paris), L. Bénichou (Paris)
}

Introduction : Devenue incontournable de nos jours pour la réhabilitation de patients présentant des édentements unitaires, partiels ou totaux, la chirurgie implantaire a connu ces dernières années de nombreuses évolutions. Même si les mesures radiographiques et l'analyse des modèles d'étude physiques sont toujours les ressources les plus utilisées par les praticiens pour recueillir les différentes données nécessaires à la prise en charge du patient, la révolution numérique et l'avènement de l'impression en trois dimensions (3D) ont récemment beaucoup fait évoluer les pratiques et offrent de nouveaux horizons. C'est dans cet esprit qu'une chaine méthodologique complétement numérique a été mise en place pour la création de guides chirurgicaux implantaires à l'aide d'une imprimante 3D. OBSERVATION : L'auteur évoquera les différents éléments utilisés dans le service nécessaires à la mise en place de cette chaine digitale (Cone Beam, caméra d'empreintes optiques intra-orale, logiciel de planification, imprimante 3D) avant de présenter différents cas réalisés à l'aide de celle-ci.

DISCUSSION : L'utilisation d'une telle chaine dans un service hospitalier n'est pas simple et son impact difficile à évaluer. L'auteur reviendra donc dans un premier temps sur les différents problèmes rencontrés lors de la création des différents guides chirurgicaux (un point sera notamment fait sur la législation concernant l'utilisation de ces guides imprimés en 3D dans et par un établissement hospitalier). Dans un second temps, on évaluera également l'intérêt de se doter d'une telle chaine numérique : l'intérêt financier (pour l'hôpital mais aussi pour le patient), le temps imparti à la prise en charge du patient (nombre et durée des consultations, temps dédié à la planification implantaire), et l'intérêt chirurgical (notamment l'évaluation de la précision de la thérapeutique implantaire).

CONCLUSION : Les nouvelles technologies font évoluer nos pratiques. Si elles nécessitent initialement un investissement financier et humain important (temps d'adaptation, courbe d'apprentissage ), elles permettent à terme et utilisées dans de bonnes indications de faciliter et d'améliorer la prise en charge des patients. 